\title{
Ventilación de Alta Frecuencia en Infección Respiratoria Grave por VRS
}

\author{
LETICIA YÁNEZ P. ${ }^{1}$, MICHELANGELO LAPADULA A. ${ }^{2}$, CARMEN BENAVENTE R. ${ }^{3}$, \\ BETTINA VON DESSAUER G. ${ }^{4}$, MARCOS EMILFORK S. ${ }^{5}$ \\ 1. Médico jefe de Unidad de Paciente Crítico Pediátrico de Clínica Santa María. \\ 2. Médico de Continuidad de Unidad de Paciente Crítico Pediátrico de Clínica Santa María. \\ 3. Médico Residente de Unidad de Paciente Crítico Pediátrico de Hospital Roberto del Río. \\ 4. Médico Jefe de UPCP de Hospital Roberto del Río. \\ 5. Médico jefe de Servicio de Pediatría de Clínica Santa María.
}

\begin{abstract}
High Frequency Oscillatory Ventilation in severe RSV Infection

High frequency oscillatory (HFOV) has been progressively useful in children with severe hypoxemic and hypercapnic respiratory insufficiency, at Pediatric Intensive Care Units. Objectives: To describe our experience in treatment of children with severe RSV infection unresponsive to conventional ventilation (CV), to describe effectiveness in gas exchange and to evaluate the safety of the method. A descriptive and prospective study set at two paediatric intensive care units, in Hospital Roberto del Río and Clínica Santa María of Santiago, Chile between January 2001 and December 2004. Results: 36 patients between 0.5 and 10 months of age with severe RSV infection and respiratory failure unresponsive to mechanical ventilation were connected to HFOV after an average time of conventional ventilation of 2.6 days. Arterial $\mathrm{pH}$ improved during the first hour in HFOV from 7.18 to $>7.3$. Initial $\mathrm{PaO}_{2} / \mathrm{FiO}_{2}$ in average was 104.8 , there was a slow and progressive improvement until 72 hours (138). $\mathrm{FiO}_{2}$ diminished since an average of $74 \%$ till $63.6 \%$ at 72 hours $\mathrm{pCO}_{2}$ went down from an average of $88 \mathrm{~mm}$ de $\mathrm{Hg}$ pre HFOV to $50 \mathrm{mmHg}$ at 12 hours, remaining low. Oxygenation index, preHFOV, was in average 18 and it maintained in 17 at 72 hours, with a $\mathrm{FiO}_{2}<60 \%$. Patients were hold in $\mathrm{HFOV}$ between 2-18 days. $3 / 36$ patients died after $1.5,4$ and 18 days of HFOV. 8,3\% presented air leak. One of these patients is oxygen dependent after 12 months of following. Conclusions: High frequency oscillatory ventilation is a useful method in the management of acute respiratory failure in children with severe infection for RSV unresponsive to conventional ventilation. It can improve gas exchange slowly and progressively with a low risk of air leak, secuelae and death.
\end{abstract}

(Key words: RSV infection, respiratory inssuficiency, high frequency, oscillatory ventilation).

Rev Chil Pediatr 2010; 81 (3): 221-227

Trabajo recibido el 16 de noviembre de 2009, devuelto para corregir el 12 de febrero de 2010, segunda versión el 15 de marzo de 2010, aceptado para publicación el 05 de abril de 2010.

Proyecto Fondecyt 1080166.

Correspondencia a:

Dra. Leticia Yánez P.

E-mail: lyanez@csm.cl 


\section{RESUMEN}

La ventilación de alta frecuencia oscilatoria (VAFO) es un modo ventilatorio cada vez más utilizado en el manejo del paciente pediátrico con insuficiencia respiratoria grave, tanto hipoxémica como hipercápnica, en unidades de paciente crítico. Objetivos. Describir nuestra experiencia en el uso de VAFO en niños con infección respiratoria aguda grave por Virus Respiratorio Sincicial (VRS), su efectividad en el intercambio gaseoso y la seguridad del método. Método: Estudio descriptivo, retrospectivo, realizado en unidades de cuidado intensivo pediátricos de Hospital Roberto del Río y Clínica Santa María de Santiago, Chile, entre los meses de Enero 2001 y Diciembre 2004. Resultados: Se conectaron a VAFO 36 pacientes de 0,5 a 10 meses de edad con falla respiratoria aguda por infección por VRS y sin respuesta a $\mathrm{VMC}$, luego de un promedio de 2,6 días. $\mathrm{El} \mathrm{pH}$ arterial mejora durante la primera hora en VAFO, de 7,18 a 7,3. $\mathrm{PaO}_{2} / \mathrm{FiO}_{2}$ inicial promedio fue de 104,8, se observó una lenta y progresiva mejoría hasta las 72 horas (138). $\mathrm{FiO}_{2}$ disminuyó de $74 \%$ hasta $63,6 \%$ en las

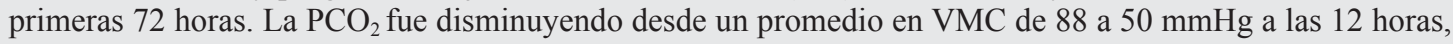
permaneciendo luego en rangos bajos. El índice de oxigenación pre VAFO, fue en promedio 18 y se mantuvo en 17 a las 72 horas de conexión, con una $\mathrm{FiO}_{2}<60 \%$. El tiempo de VAFO fue de 2-18 días. 3/36 pacientes fallecieron a los 1,5, 4 y 18 días de VAFO, 8,3\% presentaron escape aéreo. 1/36 paciente es oxígeno dependiente luego de 12 meses de seguimiento. Conclusiones: VAFO se constituyó en un método eficaz en el manejo de la falla respiratoria aguda en niños con una infección grave por VRS, que no respondieron a la VMC. Podría mejorar rápidamente la hipercapnia, y la oxigenación lenta y progresivamente, con un bajo riesgo de escape aéreo, secuelas y mortalidad.

(Palabras clave: Infección por VRS, insuficiencia respiratoria, ventilación mecánica de alta frecuencia).

Rev Chil Pediatr 2010; 81 (3): 221-227

\section{Introducción}

El virus respiratorio sincicial (VRS) es la causa principal de infecciones del tracto respiratorio inferior en lactantes ${ }^{1}$. La mayoría de los niños han sido infectados con VRS a los dos años de vida. La infección inicial es típicamente más severa y la mayoría compromete el tracto respiratorio inferior, siendo más grave y requiriendo hospitalización en los $<3$ meses $^{2}$. La forma más frecuente de presentación es la bronquiolitis aguda caracterizada por obstrucción bronquiolar y atrapamiento aéreo. Menos frecuentemente el VRS se manifiesta como neumonía y puede progresar a SDRA ${ }^{3}$. La proporción de niños que necesitan apoyo ventilatorio varía entre 8 y $21 \%$. El riesgo aumenta si existe una cardiopatía o daño pulmonar previos ${ }^{4-6}$. Los pacientes con VRS y falla respiratoria tienen obstrucción de la vía aérea pequeña o compromiso parenquimatoso restrictivo severo ${ }^{7}$ que comprometen el intercambio gaseoso, siendo el objetivo del manejo alcanzar las demandas metabólicas del paciente sin producir un daño pulmonar secundario. La ventilación mecáni- ca puede contribuir al daño primario a través del volutrauma, barotrauma, atelectrauma y biotrauma ${ }^{8}$. Una ventilación protectora busca maximizar el reclutamiento alveolar evitando la distensión a través de volúmenes corrientes pequeños y mantener abierta la vía aérea pequeña con PEEP óptimos (concepto de open lung $)^{9,10}$. El uso de PEEP en bronquiolitis es útil al reducir el PEEP intrínseco y mejorar la resistencia, permitiendo un adecuado intercambio gaseoso ${ }^{11}$.

En VAFO el volumen corriente generado ( 1 a $3 \mathrm{ml} / \mathrm{kg}$ ) es menor que el espacio muerto anatómico, a través de lo cual es posible disminuir el volumen inspiratorio al final de la inspiración, previniendo el volutrauma; además cuenta con mecanismos de intercambio que contribuyen a la oxigenación y remoción del $\mathrm{CO}_{2}$ desde el alvéolo ${ }^{12}$.

En pacientes en VMC, en los cuales es imposible mantener un intercambio gaseoso a pesar de presiones adecuadas (presiones inspiratorias hasta $30 \mathrm{~cm}$ de $\mathrm{H}_{2} \mathrm{O}$ ) y presiones espiratorias que permitan anular PEEP intrínseco que no logran movilizar volúmenes corrientes de 6 
a $8 \mathrm{ml} / \mathrm{kg}$, con aumento del riesgo de volutrauma y barotrauma entre otras, VAFO permitiría una adecuada ventilación alveolar con mínima variación de presiones alveolares, lograría reclutamiento alveolar evitando sobredistensión de zonas pulmonares no dependientes complacientes, constituyéndose en un procedimiento menos traumático de reclutamiento y estabilización pulmonar, en espera de regresión del proceso patológico original o causante.

Presentamos a continuación nuestra experiencia de manejo de enfermedad severa por VRS con ventilación de alta frecuencia oscilatoria.

\section{Pacientes y Método}

Estudio descriptivo, retrospectivo. Se revisaron las fichas médicas de 36 pacientes con infección severa por VRS con falla respiratoria aguda que no respondían a ventilación mecánica convencional y que requirieron VAFO, entre enero 2001 a diciembre 2004, en las Unidades de Cuidado Intensivo del Hospital Roberto del Río y Clínica Santa María, Santiago de Chile.

Se definió falla respiratoria que no responde a VMC como: $\mathrm{PaO}_{2} / \mathrm{FiO}_{2}$ menor de 150 y/o $\mathrm{pCO}_{2}$ mayor de $60 \mathrm{~mm} \mathrm{Hg}$ y/o índice de oxigenación mayor a 15 , con presión media de la vía aérea (PmVA) en VMC mayor a $14 \mathrm{~cm}$ de $\mathrm{H}_{2} \mathrm{O}$ y/o $\mathrm{pH}$ arterial menor de 7,25, y PEEP entre 8 a $10 \mathrm{~cm} \mathrm{H}_{2} \mathrm{O}$. Las variables edad, sexo, peso, prematuridad y enfermedad subyacente fueron consignadas, se registraron pre VAFO, a la $1,6,12,24,36,48,72,96$ y 120 horas, las variables $\mathrm{PmVA}, \mathrm{FiO}_{2}, \mathrm{PaO}_{2} / \mathrm{FiO}_{2}, \mathrm{pH}, \mathrm{pCO}_{2}$ e índice de oxigenación ( $\mathrm{PmVA} \times \mathrm{FiO}_{2} / \mathrm{PaO}_{2}$ $\mathrm{x}$ 100).

A todos los pacientes se les instaló catéter venoso central (CVC) yugular o femoral ante la necesidad eventual de algún tipo de soporte vasoactivo; a todos se realizó estudio ecocardiográfico, antes o durante el período de VAFO en busca de evidencia de hipertensión pulmonar (HTP) y/o disfunción miocárdica que requiriese necesidad de soporte vasoactivo.

Todos los pacientes fueron sedados con midazolam $0,05-0,1 \mathrm{mg} / \mathrm{kg} / \mathrm{hr}$, morfina 40-80 $\mathrm{ug} / \mathrm{kg} / \mathrm{hr}$, vecuronio $0,1 \mathrm{mg} / \mathrm{kg} / \mathrm{hr}$ en infusión continua. Este último se utilizó en algunos pacientes durante las primeras 24 horas, pero que no fue considerado si el paciente respiraba en forma confortable en VAFO.

Los pacientes fueron cambiados de la posición prona a supina entre 3 a 4 veces en el día, recibieron apoyo kinésico respiratorio 3 a 4 veces al día y aspiración de secreciones con sistema cerrado también entre 3 a 4 veces en el día o según necesidad, de acuerdo a protocolo preestablecido.

Soporte ventilatorio. Todos los pacientes fueron inicialmente manejados con ventilación mecánica convencional, en el modo presión controlada ciclada por tiempo, llegando a $\mathrm{FiO}_{2}$ $100 \%$. Se entregó VAFO por medio del ventilador Sensor Medics 3100 A, tanto en niños como en recién nacidos. Programación inicial de la PmVA fue definida como 4 puntos sobre el valor usado de PmVA en ventilación convencional $\left(17\right.$ a $25 \mathrm{~cm}$ de $\mathrm{H}_{2} \mathrm{O}$ ). Frecuencia respiratoria inicial, fue programada entre 8 a $10 \mathrm{~Hz}$, según edad del paciente, fracción inspiratoria de 0,33 , amplitud de 45 a $55 \mathrm{~cm} \mathrm{H}_{2} \mathrm{O}$ y $\mathrm{FiO}_{2}$ entre 70 y $100 \%$. Los ajustes de PmVA estuvieron basados en la saturometría de pulso en la primera hora, para lograr un $90 \%$ a $92 \%$ de saturación de hemoglobina. Radiografía de tórax debía mostrar 9 espacios intercostales, siendo el objetivo el mantener el reclutamiento alveolar y encontrar la PmVA necesaria para mantenerlos abiertos sin sobre distensión.

Se realizó ajustes en la amplitud para mantener vibración torácica y $\mathrm{pCO}_{2}$ que permitiese mantener $\mathrm{pH}$ sobre 7,2. Una vez que el pulmón hubo sido reclutado y la $\mathrm{FiO}_{2}$ fue igual o menor a $60 \%$, comenzamos con una lenta pero cuidadosa reducción de la PmVA, disminuyendo entre 0,5 y 1 punto cada vez, según radiografía de tórax y saturación, siendo cautelosos en la mantención de un "pulmón abierto". Si la $\mathrm{pCO}_{2}$ se elevaba se aspiraban secreciones, se ajusta el necesario Delta $\mathrm{P}$ y se regresa a la PmVA necesaria para lograr reclutamiento.

Definimos Hipertensión Pulmonar (HTP) ecocardiográficamente como la presencia de regurgitación tricuspídea y según gradiente trans pulmonar.

El pronóstico fue consignado en términos de 
sobrevida y complicaciones. Estas fueron definidas como: a) fuga aérea: enfisema sub cutáneo, neumomediastino o neumotórax; b) Dependencia del oxígeno, definida como la necesidad de oxígeno entre 0,5 y 2 lt por minuto al momento del alta hospitalaria.

\section{Resultados}

La Unidad de Paciente Crítico Pediátrico (UPCP) del Hospital Roberto del Río tiene 18 camas, con un promedio de admisiones durante el período de estudio de 840 pacientes, 39,3\% fueron ventilados invasivamente; la UPCP de Clínica Santa María tiene 8 camas, con un promedio de ingresos de 540 pacientes. Entre 2001 y 2004, del total de ingresos, $30,8 \%$ fueron ventilados mecánicamente.

Durante el período de estudio 64 pacientes fueron ventilados con VAFO por distintas causas. Todos ellos cumplieron los criterios de ingreso a VAFO; 36/64 presentaban infección respiratoria aguda grave por VRS. Todos los pacientes recibieron ventilación mecánica convencional previa.

Las características clínicas de nuestra serie está descrita en la tabla 1 . Todos tenían neumonía y 29/36 presentaron sindrome bronquial obstructivo (SBO) severo. Dos tenían daño pulmonar crónico y $2 / 36$ presentaron neumotórax antes de ingresar a VAFO.

Los pacientes estuvieron hospitalizados en UCI previo al ingreso a VAFO en promedio 3 días. El promedio de días en ventilación mecánica convencional previa, fue de 2,6 (1-15 días). La permanencia promedio en VAFO fue de 6 días (2-18 días); 12/36 pacientes presentaron HTP por Ecocardiografía.

Se decidió ingreso a VAFO con índice de oxigenación entre 7 y 40, promedio de 17, aquellos pacientes que ingresaron a VAFO con $\mathrm{IO}<15$, todos tenían $\mathrm{pCO}_{2}$ mayor de $80 \mathrm{mmHg}$ (promedio $106 \mathrm{mmHg}$ ). El 83\% de los pa-
Tabla 1. Características clínicas de pacientes con infección por VRS en VAFO

\begin{tabular}{|lll|}
\hline Antecedente & Número/Rango & Mediana/Promedio \\
Sexo & 15 mujeres/21 hombres & \\
Edad & 15 días-10 meses & Mediana: 2,5 meses \\
Peso & $2240-8940 \mathrm{gr}$ & Promedio: $5491 \mathrm{gr}$ \\
$\begin{array}{l}\text { Antecedente } \\
\text { de prematurez }\end{array}$ & $13 \mathrm{pac} / 27-37 \mathrm{sem}$ & Mediana: $33 \mathrm{sem}$ \\
$\begin{array}{l}\text { Cardiopatía } \\
\text { congénita no } \\
\text { cianótica }\end{array}$ & $4 \mathrm{pac}$ & \\
\hline
\end{tabular}

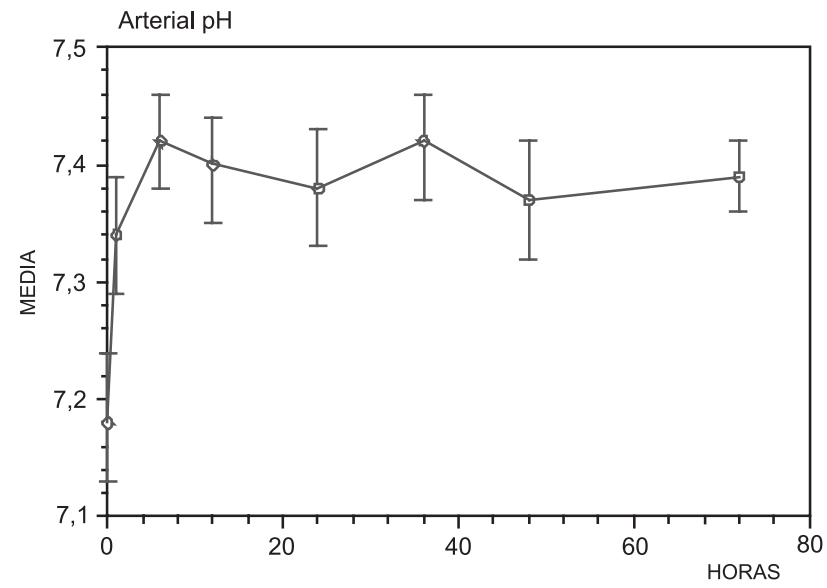

Figura 1. $\mathrm{pH}$ aumentó en forma significativa desde la primera hora en $\operatorname{VAFO}(p=0,0001)$.

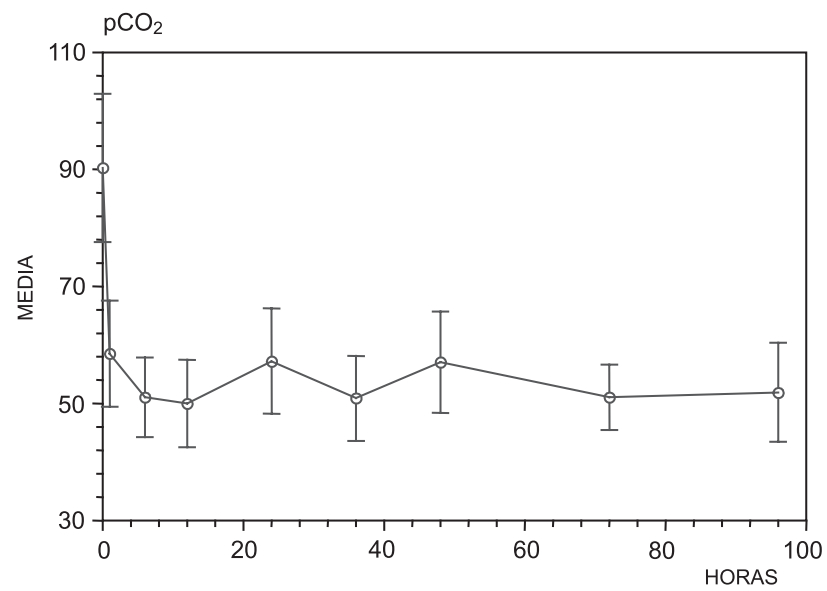

Figura 2. $\mathrm{pCO}_{2}$ disminuyó de un promedio pre VAFO de $90 \mathrm{~mm} \mathrm{Hg}$ (rango $41-149 \mathrm{~mm} \mathrm{Hg}$ ) a $50 \mathrm{mmHg}$ a las 12 horas, sin embargo esta disminución solo es significativa a las 72 horas $(\mathrm{p}=0,0001)$. 


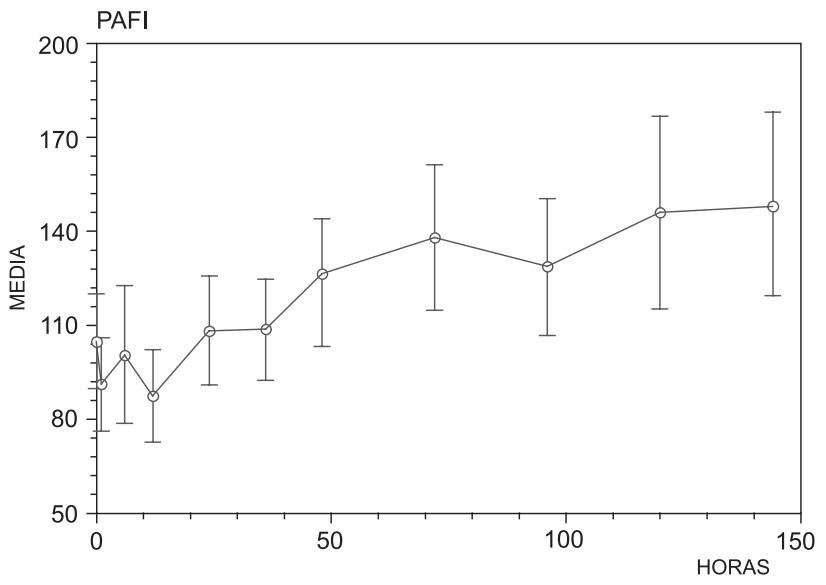

Figura 3. $\mathrm{PaO}_{2} / \mathrm{FiO}_{2}$ inicial en promedio fue 104,8 (40-200), luego de la primera hora mejora lenta y progresivamente hasta 120 a las 138 , siendo solo significativo el aumento a las 120 horas $(\mathrm{p}=0,008)$.

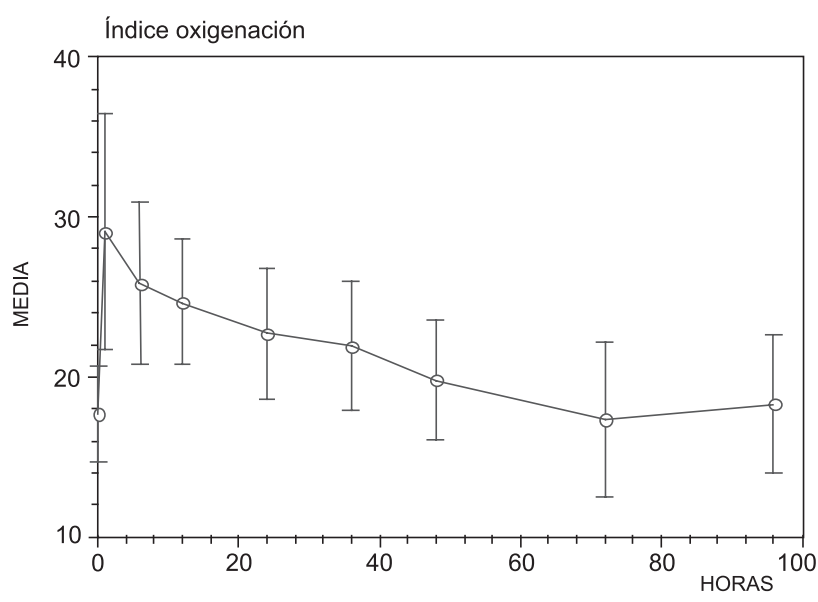

Figura 4. El índice de oxigenación preVAFO se mantiene en 17 a las 72 horas, pero con una $\mathrm{FiO}_{2}<60 \%$.

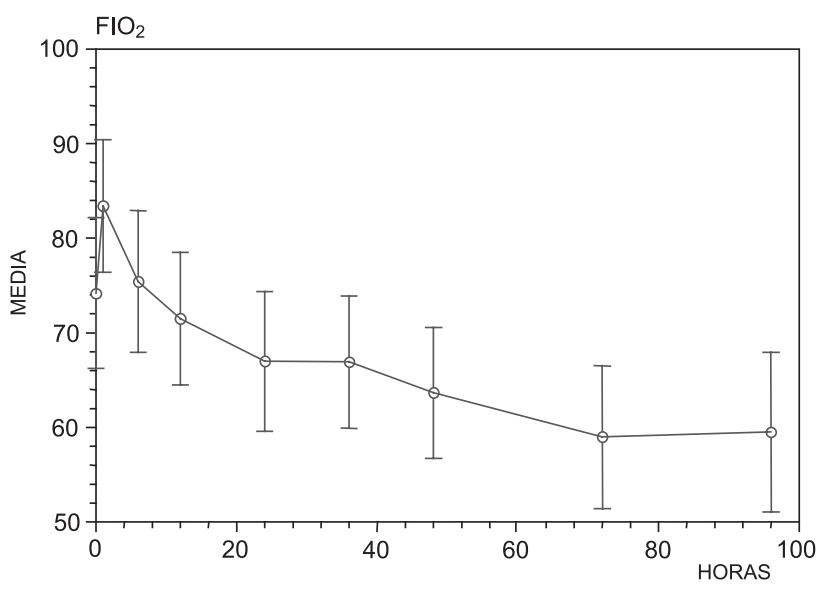

Figura 5. $\mathrm{FiO}_{2}$ disminuyó progresivamente desde un promedio de $74 \%$ a $<70 \%$ desde las 24 horas, llegando a $58 \%$ a las 72 horas en forma significativa $(\mathrm{p}=0,0064)$. cientes tenía $\mathrm{pCO}_{2}$ mayor de $60 \mathrm{~mm} \mathrm{Hg}$. La PmVA va entre 10 y $14 \mathrm{~cm}$ de $\mathrm{H}_{2} \mathrm{O}$, con un promedio de $14 \mathrm{~cm}$ de $\mathrm{H}_{2} \mathrm{O}$ y en la primera hora en VAFO se mantuvo entre 16 y $25 \mathrm{~cm}$ de $\mathrm{H}_{2} \mathrm{O}$, con un promedio de $20 \mathrm{~cm}$ de $\mathrm{H}_{2} \mathrm{O}$, posteriormente se mantuvo esta presión hasta iniciar su descenso entre las 24 a 36 horas.

Intercambio gaseoso. La mejoría de la acidosis respiratoria después de la primera hora en VAFO gracias a una reducción efectiva de la $\mathrm{pCO}_{2}$ es significativa desde las primeras horas de conexión a VAFO (figuras 1 y 2). La oxigenación aumenta en forma lenta, pero progresiva (figuras 3, 4 y 5). Por las características del trabajo no se puede relacionar directamente esta mejoría con VAFO o con evolución de la enfermedad, pero permitió mantener a los pacientes sin aumentar morbilidad.

Complicaciones y mortalidad. 3/36 presentaron neumotórax en VAFO, todos menores de 7 meses (rango de $1 \mathrm{a}$ 7 meses), dos de ellos Prematuros menores de 34 semanas, ninguno falleció. Uno de los pacientes desarrolló Enterocolitis Necrotizante secundaria a hipoxemia severa al séptimo día de evolución. La mortalidad de nuestra serie fue 3/36, a los 1,5-4-18 días de evolución. Todos ellos presentaban hipertensión pulmonar, un paciente era portador de cardiopatía congénita no cianótica (Comunicación interauricular del lactante).

Un lactante, de 6 meses de edad, con antecedente de Recién nacido de pre término 25 semanas, quien permaneció 38 días hospitalizado, 33 de ellos en UCI, con 18 días de ventilación mecánica convencional y 12 en VAFO, fue dado de alta con dependencia de oxígeno, y $15 / 36$ presentaron bronquitis obstructiva recidivante, en el seguimiento a $6 \mathrm{y}$ 12 meses.

\section{Discusión}

En esta serie se evidencia lo descrito en la literatura, en cuanto a que la in- 
fección por VRS es frecuente en menores de 2 años y más severa en los menores de 3 meses $^{2}$.

Nuestros resultados muestran que VAFO mejora lentamente la oxigenación y rápidamente la hipercapnia en pacientes menores de 12 meses con infección por VRS incluidos aquellos con antecedente de prematurez y cardiopatía.

Arnold y col ${ }^{13}$, condujeron el primer estudio prospectivo, aleatorizado que comparaba VAFO y VMC en niños con falla respiratoria con $\mathrm{IO}<13$, encontrando que una estrategia agresiva de reclutamiento pulmonar, con presiones medias de vía aérea mayores mejoraba en forma significativa la oxigenación sin aumentar el riesgo de barotrauma.

Hasta ahora no hay estudios comparativos entre VAFO y ventilación mecánica convencional que apoyen el uso de VAFO en VRS, pero sí existen series pediátricas que han usado VAFO cuando la falla respiratoria no ha respondido a ventilación mecánica convencional y han observado mejoría de la oxigenación y la acidosis respiratoria. Medbo y col, ingresaron a cuatro lactantes menores de 6 meses con infección por VRS que requerían ventilación mecánica a VAFO, todos sobrevivieron, aparentemente sin secuelas ${ }^{14}$. Jaballah y col ${ }^{15}$, usaron VAFO en 20 pacientes con falla respiratoria y enfermedad alveolar difusa y mejoraron oxigenación e hipercapnia, dos de ellos tenían infección por VRS, también respondieron en forma favorable.

Sarnaik cambió a VAFO a 31 pacientes con falla respiratoria severa a pesar de $\mathrm{VMC}^{16}$, de los cuales 13 tenían bronquiolitis por VRS, logrando mejoría en la oxigenación y una rápida disminución de la $\mathrm{pCO}_{2}$, probablemente gracias a la falta de alternancia entre períodos de inflación y deflación que contribuyen al volutrauma y atelectrauma, evitando una sobredistensión alveolar y favoreciendo una mejor ventilación ${ }^{17}$.

Más de la mitad de nuestros pacientes tenían obstrucción de vía aérea con el consecuente riesgo de atrapamiento aéreo, sin embargo, esta complicación fue baja, esto se podría explicar por PmVA necesarias para mantener abierta la vía aérea además de favorecer el reclutamiento alveolar, tal como se describe en otra experiencia de 9 pacientes con bronquiolitis por VRS manejados con VAFO, que mejoraron oxigenación sin presentar fallecidos ni complicaciones ni enfermedad residual pulmonar $^{18}$. Esto también sería apoyado por la gran incidencia de hipercapnia en nuestro grupo con una rápida disminución al ser conectados a VAFO.

Un delta $\mathrm{P}$ bajo, el mínimo necesario para lograr una adecuada vibración evitaría una mayor hiperinsuflación al mantener volúmenes corrientes bajos, especialmente en aquellos pacientes obstructivos. Pensamos que esta estrategia ventilatoria fue muy importante en la mejoría de la oxigenación y ventilación previniendo el atrapamiento aéreo y las escasas complicaciones que se observan en nuestro estudio.

Los resultados obtenidos, sugieren que es muy importante el uso precoz de este método ventilatorio, basados tanto en la oxigenación como ventilación, no sólo como medida de salvataje, ya que puede proveer un adecuado intercambio gaseoso manteniendo un volumen pulmonar elevado al final de la espiración, sin inducir sobredistensión, lo que se manifestaría en una disminución del daño pulmonar por ventilación mecánica.

La mortalidad global por VRS grave en esta serie de casos es baja, probablemente no atribuible directamente a la ventilación, relacionándose con hipertensión pulmonar y cardiopatía congénita. En el grupo de Sarnaik y col, en que fallecieron 4 de los pacientes con VRS, destaca la estrategia poco agresiva ya que iniciaron la VAFO con PMVA semejantes a las que tenían en VM convencional y luego las fueron adecuando según oxigenación arterial, en este mismo estudio relacionan una tardanza en la mejoría del índice de oxigenación antes de 6 horas con mayor riesgo de mortalidad, quedaría por definir si esto no se relacionaría con poca agresividad en el momento de conectar al paciente a VAFO.

Concluimos que la ventilación de alta frecuencia oscilatoria sería una buena alternativa en el manejo de pacientes con falla respiratoria secundaria a VRS en cualquiera de sus manifestaciones, que no responde a ventilación mecánica convencional. Sin embargo son nece- 
sarios estudios aleatorizados controlados para confirmar estos beneficios.

\section{Referencias}

1.- Hall $C B$ : Respiratory syncytial virus and parainfluenza virus. N Engl J Med 2001; 344: 1917-28.

2.- Glezen WP, Taber LH, Frank AL, Kasel JA: Risk of primary infection and reinfection with respiratory syncytial virus. Am J Dis Child. 1986; 140: 543-6.

3.- Frankel LR, Derish MT: Respiratory syncytial-virus induced respiratory failure in the pediatric patient. New Horiz 1999; 7: 335-46.

4.- Shay DK, Holman RC, Newman RD, et al: Bronchiolitis-associated hospitalizations among US children, 1980-1996. JAMA 1999; 282: 1440-6.

5.- Wang EEL MD, CM, Law BJ MD: Pediatric Investigators Collaborative Network on Infections in Canada (PICNIC) study of admission and management variation in patients hospitalized with respiratory syncytial viral lower respiratory tract infection J Ped 1996; 129: 39039.

6.- Behrendt CE, Decker MD, Burch DJ, et al: International variation in the management of infants hospitalized with respiratory syncytial virus. International RSV Study Group. Eur J Pediatr 1998; 157: 215-20.

7.- Hammer J, Numa A, Newth C: Acute respiratory distress syndrome caused by respiratory syncytial virus. Pediatr Pulmonol 1997; 23: 176-83.

8.- Dreyfuss D, Saumon G: Ventilator induced lung injury: Lessons from experimental studies. Am J Respir Crit Care Med 1998; 157: 294-323.

9.- The Acute Respiratory Distress Syndrome Network: Ventilation with lower tidal volumes as compared with traditional tidal volumes for acute lung injury and the acute respiratory distress syndrome. $\mathrm{N}$ Engl $\mathrm{J}$ Med 2000; 342: 1301-8.

10.- Amato MB, Barbas CS, Medeiros DM: Effect of a protective-ventilation strategy on mortality in the acute respiratory distress syndrome. N Engl J Med 1998; 338: 347-54.

11.- Newth C, Hammer J: Pulmonary function in ventilated infants with bronchiolitis. Pediatr Pulmonol 1998; 26: 438-9.

12.- Pillow JJ MBBS, FRACP, PhD: High-frequency oscillatory ventilation: Mechanisms of gas exchange and lung mechanics, Crit Care Med 2005; 33, No. 3 (Suppl).

13.- Arnold JH MD, Hanson JH, Toro Figuero LO, et al: Prospective randomized comparison of high-frequency oscillatory ventilation and conventional mechanical ventilation in pediatric respiratory failure Crit Care Med 1994; 22: 1530-9.

14.- Medbo S, Finne PH: Respiratory syncytial virus pneumonia ventilated with high frequency oscillatory ventilation Acta Paediatr 1997; 86: 766-7.

15.- Jaballah $N B M D$, Khaldi $A M D$, Mnif $K M D$ : Highfrequency ventilation in acute pediatric respiratory failure. Ped Crit Care Med 2006; 7: 362-7.

16.- Sarnaik AP MD FCCM, Meert KL MD: Predicting outcome in children with severe acute respiratory failure treated with high-frequency ventilation. Crit Care Med 1996; 24: 1396-42.

17.- Arnold JH MD: High-frequency ventilation in the pediatric intensive care unit Ped Crit Care Med 2000; 1 (2) 93-9.

18.- Duval E, van Vught AJ, Leroy PLJM: High-frequency oscillatory ventilation in bronchiolitis. Respir Med 1999; 93 (6): 435-40. 